\title{
Wireless Power Transfer in a Body-Centric Setup: Recent Advances and Remaining Challenges
}

\author{
Hendrik Rogier, Sam Agneessens, Thomas Cuyckens, Sam Lemey, Peter Vanveerdeghem, \\ Luigi Vallozzi, Patrick Van Torre \\ Dept. of Information Technology, Ghent University/iMinds, 9000 Ghent, Belgium, \\ hendrik.rogier@UGent.be
}

In the recent past, wireless power transfer (WPT) has attracted a lot of research interest as enabling technology for wireless sensors networks, within the context of the Internet of Things, and to finally achieve fully autonomous electronic devices. A setup where wirelessly powered electronic devices are deployed on the human body is both very appealing and very challenging. Indeed, autonomous sensors that are unobtrusively integrated into the wearer's jacket may monitor body parameters and the user's environment while remaining invisible to the mobile user as well as to other persons in his/her proximity. Unfortunately, the bodycentric environment is one of the hardest configurations to implement such a wireless transfer, given the movement of the wearers and the potential health hazards associated to the exposure to radiofrequency (RF) fields.

To ensure the mobility of the person when powering the wearable electronic system, the most practical solution is to implement WPT in the far field. Yet, in this setup it is difficult to reach high transfer efficiencies to body-worn antennas that constantly change orientation. Since regulations in terms of specific absorption rates must be satisfied at all time to avoid health risks, the rectifying circuit at the receiver will have to deal with very large voltage levels. In this presentation, we will discuss a paradigm that might increase the overall efficiency of body-centric WPT without violating the RF field exposure regulations. The comfortable integration of multiple textile antennas into a garment may drastically increase the aperture over which RF energy can be harvested, without disturbing the movements of the mobile user. Moreover, a clever distribution of the wearable antennas over the complete area of a jacket may also mitigate the fluctuations in harvested RF energy due to movement. To optimize the wireless transfer of energy within the exposure limits imposed by the regulatory framework, ultra-wideband signals may be utilized.

We will outline the abovementioned recent solutions for WPT in a body-centric setup. In addition, we will demonstrate how RF exposure limits may be continuously monitored in realtime by a wearable distributed exposimeter system. Moreover, we will describe a modelling and design framework to evaluate ultra-wideband WPT systems. 